\title{
A STATISTICAL ANALYSIS ON THE SYSTEM PERFORMANCE OF A BLUETOOTH LOW ENERGY INDOOR POSITIONING SYSTEM IN A 3D ENVIRONMENT
}

\author{
G.G. Haagmans ${ }^{\text {a }}$, S. Verhagen ${ }^{\text {a }}$, R.L. Voûte ${ }^{\text {bc }}$, E. Verbree ${ }^{\mathrm{d}}$ \\ ${ }^{a}$ Geoscience and Remote Sensing, Civil Engineering and Geosciences Faculty, TU Delft, the Netherlands - \\ G.G.Haagmans@student.tudelft.nl, A.A.Verhagen@tudelft.nl \\ ${ }^{\mathrm{b}}$ Vice President Consulting Geo-ICT, CGI Nederland BV, George Hintzenweg 89, 3068 AX Rotterdam, the Netherlands - \\ Robert.Voute@cgi.com \\ ${ }^{\mathrm{c}}$ Department Urbanism, Faculty of Architecture and the Built Environment, TU Delft, the Netherlands - \\ R.Voute@tudelft.nl \\ ${ }^{\mathrm{d}}$ OTB - Research for the Built Environment, Faculty of Architecture and the Built Environment, TU Delft, the Netherlands - \\ E.Verbree@tudelft.nl
}

KEY WORDS: Indoor positioning system, Bluetooth low energy, system performance, theoretical design computations, precision, optimal configuration, 3D space

\begin{abstract}
:
Since GPS tends to fail for indoor positioning purposes, alternative methods like indoor positioning systems (IPS) based on Bluetooth low energy (BLE) are developing rapidly. Generally, IPS are deployed in environments covered with obstacles such as furniture, walls, people and electronics influencing the signal propagation. The major factor influencing the system performance and to acquire optimal positioning results is the geometry of the beacons. The geometry of the beacons is limited to the available infrastructure that can be deployed (number of beacons, basestations and tags), which leads to the following challenge: Given a limited number of beacons, where should they be placed in a specified indoor environment, such that the geometry contributes to optimal positioning results? This paper aims to propose a statistical model that is able to select the optimal configuration that satisfies the user requirements in terms of precision. The model requires the definition of a chosen 3D space (in our case 7x10x6 meter), number of beacons, possible user tag locations and a performance threshold (e.g. required precision). For any given set of beacon and receiver locations, the precision, internal- and external reliability can be determined on forehand. As validation, the modeled precision has been compared with observed precision results. The measurements have been performed with an IPS of BlooLoc at a chosen set of user tag locations for a given geometric configuration. Eventually, the model is able to select the optimal geometric configuration out of millions of possible configurations based on a performance threshold (e.g. required precision).
\end{abstract}

\section{INTRODUCTION}

\subsection{Indoor positioning systems}

Since GPS is not available for indoor positioning purposes, another market emerged along with alternative methods to overcome this problem. One of the alternative methods comprises indoor positioning systems (IPS), replacing the loss of GPS signals in indoor environments. First of all, an IPS is defined by Depsey (2003) as "A system that continuously determines the real-time position of something or someone in a physical space such as in a hospital, a gymnasium, a school, etc." The real-time positions of the users of the IPS are often derived via the position of their tags or mobile devices. Subsequently, the rising market of IPS is widespread and contains many technologies that can be used for indoor positioning.

One of the indoor positioning techniques that is developing rapidly is based on Bluetooth communication. Bluetooth is a wireless technology that can be used for short-range communication between different Bluetooth enabled devices (Dursch et al., 2004). From the original Bluetooth systems emerged an enhanced Bluetooth Low Energy (BLE) system. BLE was developed as a low-power solution for control and application monitoring (Gomez et al., 2012). Due to its power-efficiency, BLE is suited for devices that run for long periods on power sources (Omre and Keeping, 2010), such as mobile phones. During this research a BLE indoor positioning system of BlooLoc (BlooLoc, 2016) will be used for experimental purposes.

Bluetooth is operating in the unlicensed $2.4 \mathrm{GHz}$ industrial, scientific and medical (ISM) radio frequency (RF) band of the electromagnetic spectrum. Additionally, Wi-Fi devices, microwaves and remote control devices use the unlicensed $2.4 \mathrm{GHz}$ ISM band as well. To reduce the interference between the different devices, Bluetooth uses a frequency-hopping spread-spectrum system (FHSS), transmitting data over different frequencies at different time intervals (Bhagwat, 2001). This is achieved by hopping to different frequencies in the ISM band and a device makes about 1600 hops per second, spaced out over $1 \mathrm{MHz}$ (Dursch et al., 2004). Just like classic Bluetooth, BLE uses frequency hopping for communication purposes (Faragher and Harle, 2014).

1.1.1 Positioning vs Localization: In an indoor environment, it can be assumed that the world is flat in itself so coordinate transformations for the curvature of the Earth are not necessary. In general, positioning refers to a position in $\mathrm{x}$ and $\mathrm{y}$ coordinates for $2 \mathrm{D}$ and $\mathrm{x}, \mathrm{y}$ and $\mathrm{z}$ coordinates for $3 \mathrm{D}$ of a person or an object. If the object moves to another position, the positioning system will assign updated coordinates from the new position to the object. However, without knowledge of the surrounding environment or infrastructure of the building, the position becomes useless and uninterpretable for systems and humans (van der Ham et al., 2016). Mautz (2012) defines localization as follows 


\begin{abstract}
"The term localization underlines that the application requires topological correctness of the sensor locations, whereas the absolute coordinate position is of minor importance."
\end{abstract}

The localization of an object or person puts an absolute coordinate position into perspective regarding their environment and can exclude areas where it is not likely for a person to be, for example on a table, or the other way around, it can include areas where persons are expected to be walking. The terms positioning and localization are closely correlated with each other within the subject of indoor positioning, but there is a clear distinction in the definitions of both terms.

\subsection{Challenge}

An IPS should be able to achieve accurate positions of the user devices in case GPS is failing. However, to cover for instance a convention center consisting of large open spaces and high ceilings, the signal propagation range of the available number of beacons is often exceeded, leading to serious issues for accurate positioning of the visitors. In such a case, the geometric constellation of the beacons is in direct relation with the precision and accuracy of the positioning results. To solve this problem and to minimize the costs, the customer wants to know in what geometry the beacons should be deployed on forehand to retrieve optimal positioning results for a given 3D space. Firstly, when deploying an IPS it has to be considered that indoor environments are often covered with obstacles such as furniture, (partition-) walls, human beings and electronics in Line-of-Sight (LOS) influencing signal propagation in different ways. The second factor is depending on the physical aspects such as signal strength, range and signal attenuation. Finally, the third- and major factor, the geometric configuration of the beacons plays a major role in acquiring optimal positioning results. The geometry of the beacons is limited by the available infrastructure that can be deployed (number of beacons, basestations and tags), which on its turn is dependent on the budget and deployment effort of the costumer. Therefore, knowledge about the geometric deployment of an IPS is required in order to obtain optimal positioning results. This leads to the following question: Given a limited number of beacons, where should they be placed in a specified indoor environment, such that the geometric configuration of the beacons provide optimal positioning results?

\subsection{Approach}

To answer the question defined in section 1.2, a statistical model, referred to as theoretical design computations, is combined with practical geometric experiments. The design computations describe a theoretical simplification of a 3D space and comprise a mathematical model that is used to determine the dilution of precision, internal- and external reliability for any given set of beacon and receiver locations. Besides the definition of a chosen 3D space (in our case 7x10x6 meter), the design computations require the number of available beacons, possible user tag locations and a performance threshold (e.g. required precision). The results of the theoretical design computations are validated by performing practical experiments with the BlooLoc IPS. The geometric configuration used in the design computations can be deployed in a real 3D space by using the IPS of BlooLoc. Subsequently, the measured precision can be compared to the modeled precision for given user tag locations and the model is validated.
Eventually, the model must be able to select the optimal geometric configuration out of millions of possible configurations based on a performance threshold (e.g. required precision).

\subsection{Outline of the paper}

This paper is subdivided in two parts. The first part 'Methodology' comprises the BlooLoc technology and information about their IPS. The model of the theoretical design computations will be elaborated together with the set-up for both the design computations and the practical experiment. The second part 'Results and discussion' contains the results and validation of the theoretical design computations, the practical experiment and finding the optimal configuration. Finally, the conclusions are drawn and some suggestions for future work are given.

\section{METHODOLOGY}

The IPS market is wide-spread and offers many different solutions for indoor positioning and localization. For this research the IPS of BlooLoc using Bluetooth Low Energy has been used.

\subsection{BlooLoc Technology}

BlooLoc is an innovative tech company headquartered in Leuven (Belgium). The term BlooLoc is a combination of the terms Bluetooth Localization and offers hardware and software for accurate indoor positioning, known as yooBee (BlooLoc, 2016). BlooLoc is mainly focused on consumer type applications and uses Received Signal Strength Indicator values (RSSI) instead of time of arrival (ToA) measurements. For this research, a starter kit of BlooLoc has been used, including 1 basestation, 15 beacons and 3 tags. The starter kit corresponds with a single Cell deployment, referring to the Cell of a single basestation which spans an area of approximately $500 \mathrm{~m}^{2}$. Since all devices serviced by a Cell should be within the range of the basestation, the range of wireless indoor connections is limited to a recommended $450 \mathrm{~m}^{2}$ (BlooLoc, 2016). Furthermore, the cost of a starter kit is $2750 €$, the update rate is $1 \mathrm{~Hz}$ and the positioning error is claimed to be less than $1 \mathrm{~m}$ for $80 \%$ of the measurements. The beacons, basestation and tags communicate with each other via radio frequency (RF) signals (Bluetooth Low Energy) spread over 40 channels within the ISM $2.4 \mathrm{GHz}$ band, each with a $2 \mathrm{MHz}$ width. More information about the functionality, infrastructure or deployment can be acquired via BlooLoc (2016).

2.1.1 Indoor positioning with BlooLoc: The tags use BLE to constantly broadcast data from their inertial sensors as advertise packages within the advertising channels of the Bluetooth band. The tag sends an omnidirectional BLE pulse which is received by both basestation and beacons with fixed locations. The basestation acts as a scanner and is constantly receiving data from the advertising channels and does not broadcast information itself, whereas the beacons both receive and broadcast data. The beacons collect the data broadcasted by the tags, measure the Received Signal Strength Indicator values and the inertial sensor data of the tags and forward it to the basestation using BLE (BlooLoc, 2016). Simultaneously, the basestation collects all data and transmits it to the Cloud server via Ethernet or Wi-Fi. The cloud server subsequently calculates the positions of the moving tags and visualizes it on a digital floorplan. Moreover, the various received signals are compared to each other to eliminated redundant information. A schematic overview of the communication is illustrated in Figure 1. 


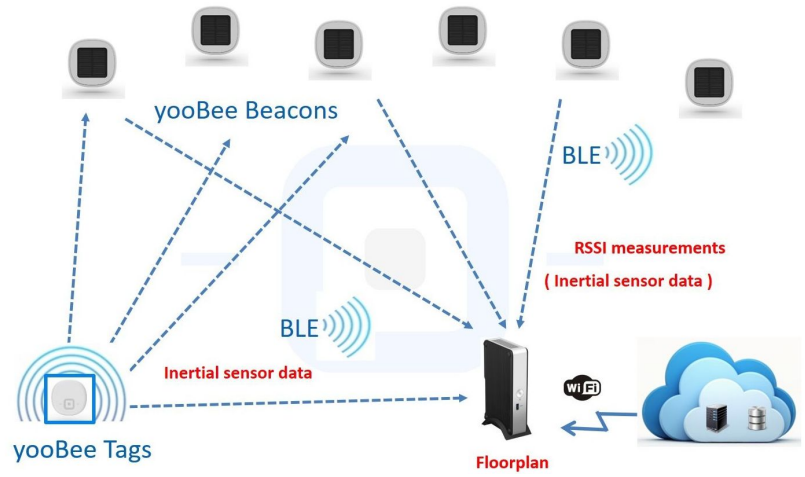

Figure 1. The communication between tags, beacons, basestation and the Cloud server. Source: BlooLoc (2016)

2.1.2 Inertial sensors and sensor fusion principle: Sensor fusion is the state-of-the-art positioning principle used by BlooLoc to compute the most likely position of a tag or mobile device (Callaerts, 2016). The principle sensor fusion comprises the fusion of a RSSI model of the environment, all past inertial sensor data and floorplan information. The inertial sensors of the tags collect information that is transmitted to the beacons. The inertial sensors of the tags contain an accelerometer, magnetometer, thermometer and a gyroscope. The RSSI model consists of all past RSSI values between each beacon and every tag or mobile device and is constantly improving and learning from historic data. Moreover, the floorplan information includes all obstacles and infrastructure of the indoor environment such as walls, corridors, doors, obstacles etc. Initially, a burn-in phase is required. Basically, the burn-in phase is a self-learning autocalibration system which over time leads to a more and more accurate positioning system by analyzing all gathered data. The more historic data, the better the RSSI model becomes and the more accurate it can account for effects of for instance shadowing and reflection. Finally, a stochastic technique based on a sequential Monte Carlo (particle filter) determines the most likely position (Callaerts, 2016).

The position information that is displayed on the floorplan contains the users orientation, a real-time position estimate $(\leq 2 \mathrm{~s}$ delay) and a delayed position estimate (16s delay) that is more accurate since it uses future data to compute the most likely position (Callaerts, 2016). If the beacons are installed right and the system has self-calibrated properly, the accuracy is claimed to be less than $1 \mathrm{~m}$ for $80 \%$ of the measurements and the update rate of the tags is $1 \mathrm{~Hz}$ which is sufficient for navigation purposes.

\subsection{System performance}

The challenges of an indoor environment come together with factors influencing the system performance, where the system performance is depending on statistical parameters such as precision and reliability. Precision is related to the repeatability or spread in outcomes of the measured values, whereas reliability is explaining the performance of statistical testing for validation and describes the ability of the observation system to check (itself) for modeling errors (Teunissen et al., 2004). The characteristics of an indoor environment (walls, obstacles, material characteristics etc.) play a major role in the disturbance of the signals. Besides the characteristics of an indoor environment, the geometry of the IPS is the key factor in whether or not a certain level of precision can be realized.
If the wavelength is long enough, RF signals can penetrate walls resulting in signal attenuation (shadowing). However, a signal can also be reflected by the wall, leading to multiple paths for a signal to travel between transmitter and receiver (multipath). Besides shadowing by walls or obstacles, a human body can cause shadowing as well, a principle called body shadowing. The RF signals are blocked by water and since an adolescent human body consists for $65 \%$ of water, persons cause attenuation of the signal as well. If the signal in the shadowing zone becomes significantly weak, the area becomes a Non-Line-of-Sight (NLOS) situation. Such a NLOS situation can be the consequence of for instance thick walls of certain material and the presence of highly reflective obstacles. In a NLOS situation, the direct path remains undetected and only multipath waves are detected (Callaerts, 2016).

The main contributor in obtaining a required precision is the Dilution of Precision (DOP). The DOP can be subdivided in many flavors, where one of the flavors is the Position Dilution of Precision (PDOP). The PDOP is the relation between the geometry of the positioning system and the measurement precision and is expressed as the square root of the diagonal of the variance matrix $Q_{\hat{x} \hat{x}}$ (see section 2.3.2). Since, the DOP is a measure reflecting on the geometric configuration of the IPS, it is very useful for determining the optimal configuration of an IPS. The DOP can be minimized for a certain user location by optimizing the geometry of the beacons within the 3D space (Spilker, 1996). To illustrate the effect of the DOP, assume a RF positioning system with two static transmitters and one mobile receiver, Figure 2.
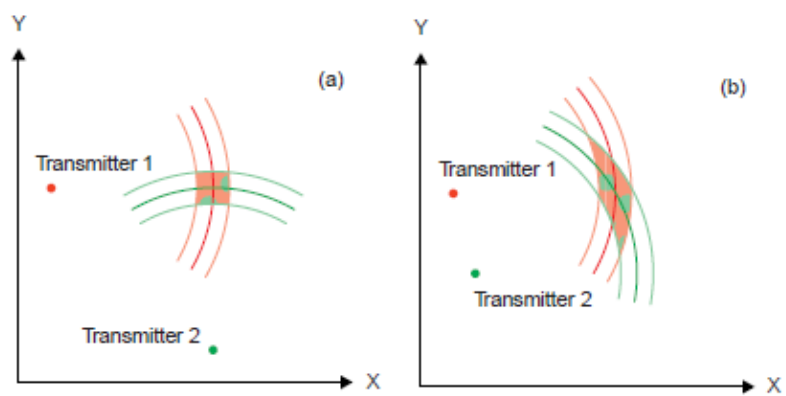

Figure 2. a) A low DOP versus b) a higher DOP due to a change in geometry. Source: Langley (1999).

Due to trilateration, the receiver is located on the intersection of the circular lines centered at the transmitters. In the first situation (Fig. 2a) the transmitters are distant with respect to each other and direction wise transmitter 1 lies orthogonal to transmitter 2. This results in $\mathrm{x}$ and $\mathrm{y}$ coordinates of the receiver with equal precision (Langley, 1999). In comparison with the second situation (Fig. 2b), the transmitters are much closer to each other and not orthogonal anymore, resulting in a larger uncertainty region and no equal precision in $\mathrm{x}$ and $\mathrm{y}$ coordinates. In this case (b) the precision is diluted in comparison to the first situation (a) as a consequence of geometry. Besides multipath, fading, NLOS, shadowing and DOP, the statistical parameters are highly dependent on range, where the range is depending on factors as signal attenuation, transmitting power, path loss and noise.

\subsection{Theoretical design computations}

The knowledge about statistical phenomena and processes that are influenced by the factors described in section 2.2 have to be understood in order to validate observational data with a theoretical model. Before acquiring observational data, the quality of the 
data can be estimated by applying design computations. The theoretical design computations will assess the quality of data with multiple hypothetical geometries and are based on existing 3D spaces. The design computations require the definition of a chosen $3 \mathrm{D}$ space (in our case $7 \times 10 \times 6$ meter), the number of available beacons, possible user tag locations and a performance threshold (e.g. required precision). For any given set of beacon and receiver locations, the dilution of precision, internal- and external reliability can be determined on forehand. Eventually, the design computations are validated with observational data acquired with the BlooLoc IPS

2.3.1 The model: A tag sends an omnidirectional BLE pulse and subsequently the pulse is intercepted by the beacons and the basestation, both with fixed locations. The beacons are intercepting the BLE pulse, gathering the data and forward it to the basestations. Eventually, the basestation calculates the distances between the tags location and every beacon based on the trilateration principle, which will lead to an estimate of the tags position. The theoretical design computations are therefore based on a system of $m$ non-linear equations, since the 3 unknown parameters are the unknown positions of the tag $\left(x_{T}, y_{T}, z_{T}\right)$. For the theoretical design computations, it is assumed that $x_{i}, y_{i}, z_{i}$ for $i=1, m$, are the known locations of the beacons. The BlooLoc set contains 15 beacons, limiting the maximum amount of available beacons to $m=15$. The unknown distance from the beacon $j$ to the tag can then be determined by $d=\sqrt{\left(x_{j}-x_{T}\right)^{2}+\left(y_{j}-y_{T}\right)^{2}+\left(z_{j}-z_{T}\right)^{2}}$. Considering the amount of beacons $i=1,, m(m \leq 15)$ the system of nonlinear equations can be expressed as

$$
\left[\begin{array}{c}
d_{1} \\
\vdots \\
d_{m}
\end{array}\right]=\left[\begin{array}{c}
\sqrt{\left(x_{1}-x_{T}\right)^{2}+\left(y_{1}-y_{T}\right)^{2}+\left(z_{1}-z_{T}\right)^{2}} \\
\vdots \\
\sqrt{\left(x_{m}-x_{T}\right)^{2}+\left(y_{m}-y_{T}\right)^{2}+\left(z_{m}-z_{T}\right)^{2}}
\end{array}\right]
$$

It has to be mentioned that this is not exactly how the BlooLoc IPS works (see section 2.1.1), but this allows the design computations to asses geometry. The non-linear functional model is defined as a simplification of the linear functional model $E(\mathrm{y})=$ $A x$ and can be written as $y \approx A(x)$. In the non-linear functional model, the solution for $x$ is chosen such that $A(x)$ is as close as possible to the given measurement vector $y$, where closeness is measured by the weighted sum of squares of the entries of $y-A(x)$ (Teunissen et al., 2004). To solve the system of non-linear equations, the model was linearized based on Taylor's theorem, where the $2^{\text {nd }}$ and higher order terms of Taylors theorem have been ignored. The linear approximation can then be written as $\Delta y_{0} \approx J * \Delta x_{0}$ with the gradient vector $J=\delta_{x} a_{i}\left(x_{0}\right)$ (Jacobian) in between $x$ and $x_{0}$.

2.3.2 Precision: After composition of the linear approximation, the Best Linear Unbiased Estimator (BLUE) is used to solve the inconsistent system of linear equations and to estimate the $\Delta \hat{x}_{0}$. The BLUE can be expressed as $\Delta \hat{x}_{0}=$ $\left(J^{T} Q_{y y}^{-1} J\right)^{-1} J^{T} Q_{y y}^{-1} \Delta y_{0}$, where the uncertainty of the observations $\Delta y_{0}$ is expressed by the $Q_{y y}$ matrix. The $Q_{y y}$ matrix is assumed to be diagonal and the observations have an initial standard deviation (std) of $\sigma=1$ meter. Furthermore, the precision is estimated by computing the variance matrix $Q_{\hat{x} \hat{x}}=\left(J^{T} Q_{y y}^{-1} J\right)^{-1}$, where the diagonal is representing the variance. Eventually, the total precision or the PDOP can be derived by

$$
\sigma_{T}=\frac{1}{\sigma} \sqrt{\sigma_{x}^{2}+\sigma_{y}^{2}+\sigma_{z}^{2}}
$$

where the standard deviations $\sigma_{x}, \sigma_{y}$ and $\sigma_{z}$ are computed by the model via the square root of the diagonal of the variance matrix $Q_{\hat{x} \hat{x}}$ and the intial standard deviation is $\sigma=1$ meter.

2.3.3 Reliability: Besides the precision, possible test results can be discussed in advance of an actual practical test by elaborating the term reliability. The reliability is a term explaining the performance of statistical testing for validation and describes the ability of the observation system to check (itself) for modeling errors (Teunissen et al., 2004). The primary data analysis, existing of a functional model and least square estimations, is able to detect disturbances, outliers and other biases. However, some errors are left undetected by the statistical testing and the reliability helps to discuss the statistical possibility that errors are left undetected during the test experiments. The reliability can be subdivided in internal- and external reliability. Generally, the theoretical design computations are able to simulate every possible user tag positions within a local $x, y, z$ grid. Therefore, the design computations can compute the total precision or PDOP from the variance matrix and both internal- and external reliability at every user tag location. This paper will mainly focus on the modeled precision, since the modeled precision is used to validate the design computations with the measured precision from the practical experiment.

\subsection{Set-up}

2.4.1 Theoretical design computations: The initial model requires the number of beacons and the size of the theoretical 3D space as input by the user. The shape of the theoretical 3D space is assumed to be a cube, since most indoor environments can be described by squared spaces. Furthermore, the locations of the beacons have to be predefined by the user such that the beacons are located within the predefined cube. The point density of the cube can vary and depends on the user requirements. In this case, the points represent possible locations for a mobile device and are used to calculate the precision, internal- and external reliability. The denser the points, the more possible locations for a mobile device and the better the estimation and statistical analysis becomes. To visualize the theoretical 3D space and the locations of the beacons, one of the possible geometric configuration including 15 beacons is illustrated in Figure 3.

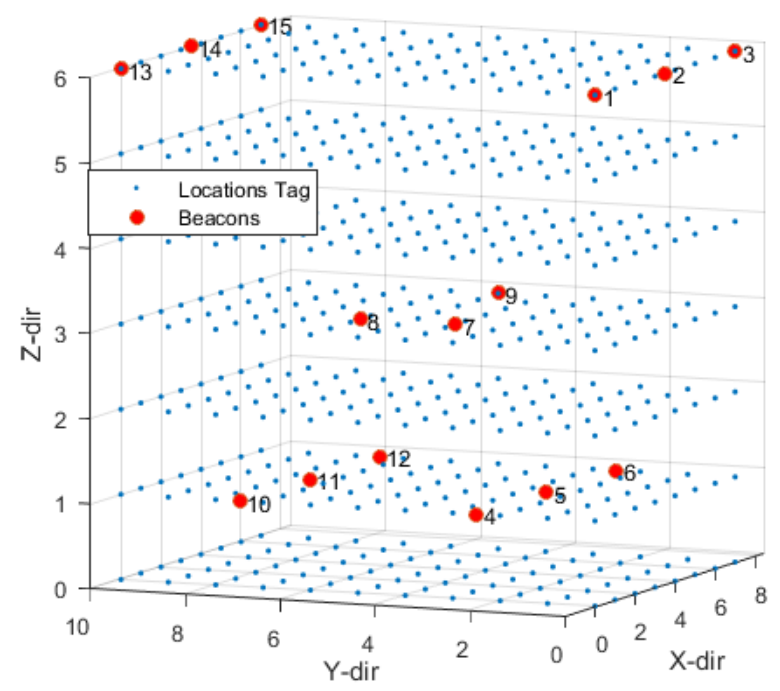

Figure 3. A theoretical 3D space of a 7x10x6 meter cube with a geometry of 15 beacons (red) and possible tag locations (blue). 
2.4.2 The practical experiment: The geometric experiments are based on the results derived from the theoretical design computations. Since the theoretical design computations provide measures for precision, internal- and external reliability for different theoretical set-ups, a validation can be done by a practical experiment. The geometry of the practical experimental set-up is based on the geometry that is tested in the theoretical design computations, such that a comparison can be made between the predicted precision and the obtained practical precision. The practical experiment was carried out in a hallway at the first floor of the CiTG building at the TU Delft. Assuming the first floor begins at $z=0$ meter, the ceiling height is $z=5.993$ meter. The $2 \mathrm{D}$ floorplan of practical experiment is illustrated in Figure 4.

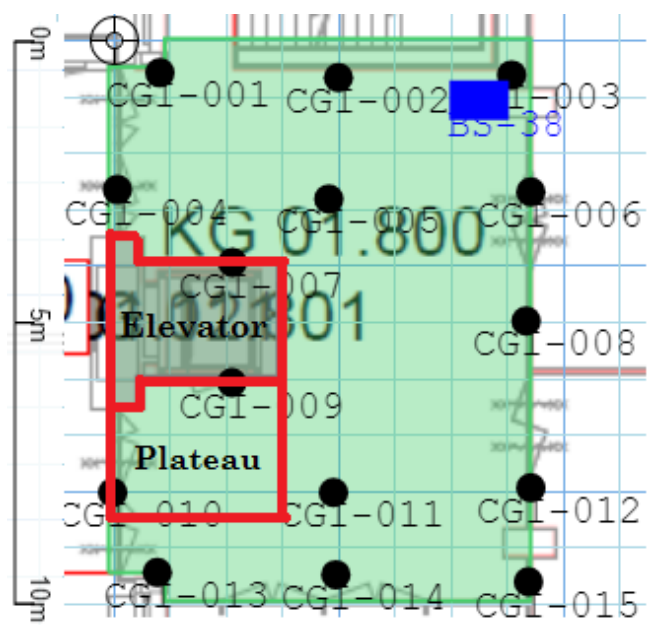

Figure 4. The test area (green) with the locations of the beacons (black), basestation (blue) and the elevator shaft (red).

The test area is approximately $7 \times 10 \times 6$ meter, equal to the $3 \mathrm{D}$ space defined in the design computations. The 15 beacons (black circles) have been deployed based on the geometric configuration of Fig. 3. Beacons 1-3 and 13-15 were deployed at the ceiling ( $z=5.993 \mathrm{~m}$ ), beacons $4-6$ and $10-12$ at $z \approx 1 \mathrm{~m}$ and beacons 7-9 in the center row at $z=3.00 \mathrm{~m}$. The measurements with the BlooLoc tag were done according to a 1 meter grid in $x$ and $y$ direction with its origin in the upper-left corner of the test area (Fig. 4). In order to prevent signal attenuation by the human body, the tag was placed on a tripod.

\section{RESULTS AND DISCUSSION}

\subsection{Design computations}

3.1.1 Precision: The total precision, internal- and external reliability have been computed for the geometric configuration of Fig. 3. The total precision is visualized in Figure 5 with a minimum of 0.7854 meter and maximum of 1.0744 meter. The precision shows a symmetrical pattern, with an increase in precision towards the center of the 3D space. Some of the tag locations towards the edges of the 3D space show a decrease in precision (higher standard deviation). The direct relationship between the precision and geometry is reflected in the center points $(x=0-7, y=3-7$ and $z=0-6)$. In these regions, the precision is high in both $\mathrm{x}, \mathrm{y}$ and $\mathrm{z}$ direction resulting in an increased total precision. In other words, the position uncertainty is smaller as a consequence of the geometry resulting in a lower dilution of precision. However, towards the edges of the 3D space, the position uncertainties become larger or the dilution of precision becomes higher. This is a consequence of the geometry which causes a decrease of precision in the $z$ direction and thus a decrease in the total precision (higher standard deviations).

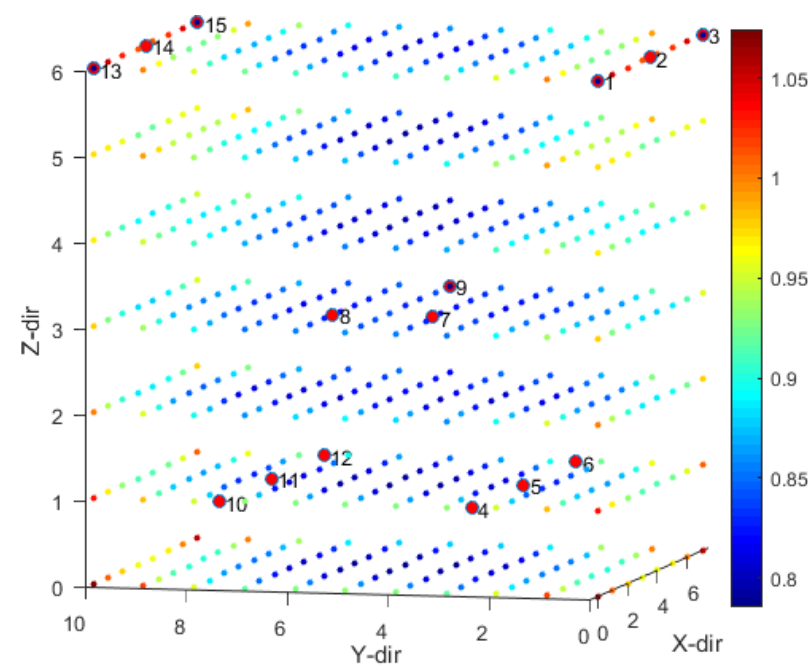

Figure 5. The total precision for possible user tag locations for the geometric configuration of 15 beacons.

\subsection{The practical experiment}

3.2.1 Position estimates: The tripod with the tag was placed on a virtual 1 by 1 meter grid and measured for at every location for 90 seconds, resulting in numerous positioning estimates at each of the, in total 46, measurement locations of Figure 6. The measurement at location 28 contained no data and has not been used for further analysis. Furthermore, the test area consists of hallway without obstacles, where the only exceptions are an elevator shaft located between $(x=0-2$ and $y=3-6$ and a big rock (Fig. 6, circle). The elevator has an exit to a plateau at a subfloor floor at $z=2.55 \mathrm{~m}$ which might cause some signal attenuation or distortion for measurements taken underneath the plateau.

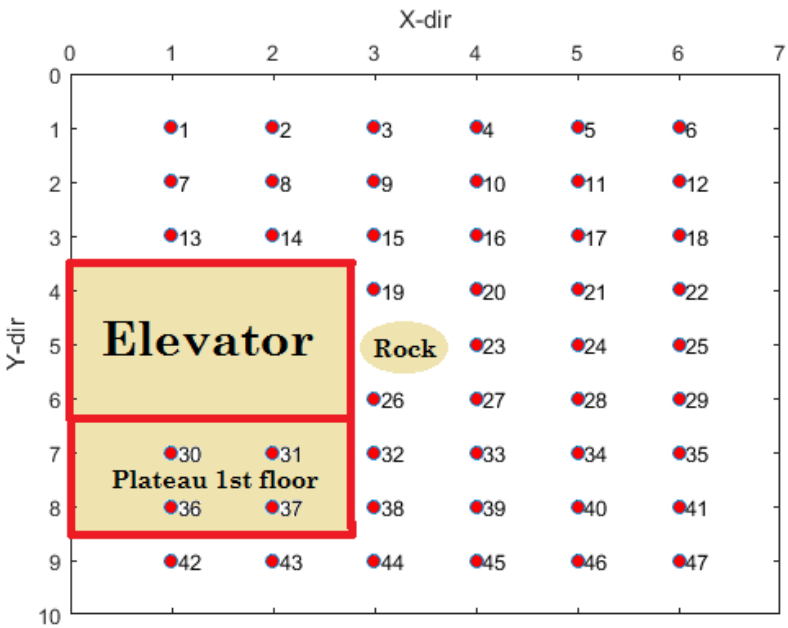

Figure 6. The measurement locations (red dots) in the test area.

For every possible tag location, the position in $x$ and $y$ coordinates was calculated by BlooLoc along with corresponding standard deviations. Eventually, the positioning estimates were averaged over all measurement locations and compared to their true locations. The distance $d$ between the true locations and the corresponding estimated positions has been used to filter out the points 
that had been estimated at $d>1.5$ meter from their true location, resulting in the removal of 19 points. Since the accuracy is a standard for the closeness of the measured values with respect to the true values, the accuracy results are visualized in Figure 7. It is hard to distinguish a pattern, since some estimates have a high accuracy or in other words, are relatively close to their true positions (nr. 2, 4, 29, 39, 43, 45), while others have a significant offset (nr. 5, 17, 21, 46, 47) and thus a low accuracy. However, it is remarkable that all points underneath the plateau at the $1^{\text {st }}$ floor have been removed due to a offset of $d>1.5$ meter. The removal of these points is most likely due to a combination of NLOS and shadowing. Most of the position estimates that are closest to their true locations are found at $x=4$. The higher offsets next to the elevator shaft are most likely caused by reflections and multipath due to the metal elevator shaft.

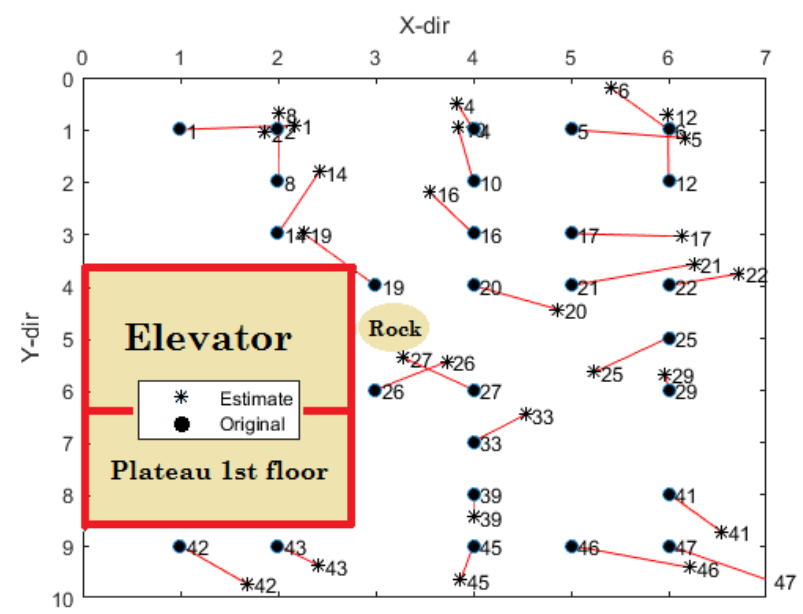

Figure 7. The accuracies of the practical results.

3.2.2 Comparison of precisions: Finally, the standard deviations of the estimated $x$ and $y$ positions were computed by the IPS. The standard deviations were used to calculate the total precision (in $x, y$ direction with equation 2) and can be compared to the precision values of the theoretical design computations at $z=1$. Similar to the position estimates, the computed total precision values were averaged over all measurement locations. For convenience, the total precision computed by the IPS has been visualized at their true locations in Figure 8, but take into account that the estimated positions differ from the true locations (Fig. 7). From Fig. 8 can be seen that the precision of the design computations ranges from $0.75-1$ meter, whereas the estimated precision of the IPS ranges from $0.75-1.4$ meter where the majority of the estimated precision lies between 0.75 and 1.1 meter.

The highest precision is found in the center of the area for both design computations as the results of the practical experiment. As expected, the precision values of the practical experiment differ slightly from the precision predicted by the design computations. Furthermore, at some places the precision values of the IPS shows a significant decrease in precision (nr. 4, 15 - 18 and 34) as a consequence of either systematic errors or due to signal reflections of the metal elevator shaft. Note that the design computations define the precision for a given geometric configuration in an ideal 3D space and do not take reflections, materialistic properties, signal attenuation etc. into account. Furthermore, the precision has only been validated at one height level $z=1$. Note that the precision experiments could be performed at any height, however the height level of $z=1$ has been chosen since most people wear mobile devices in their pockets at approximately $z=1$ meter.
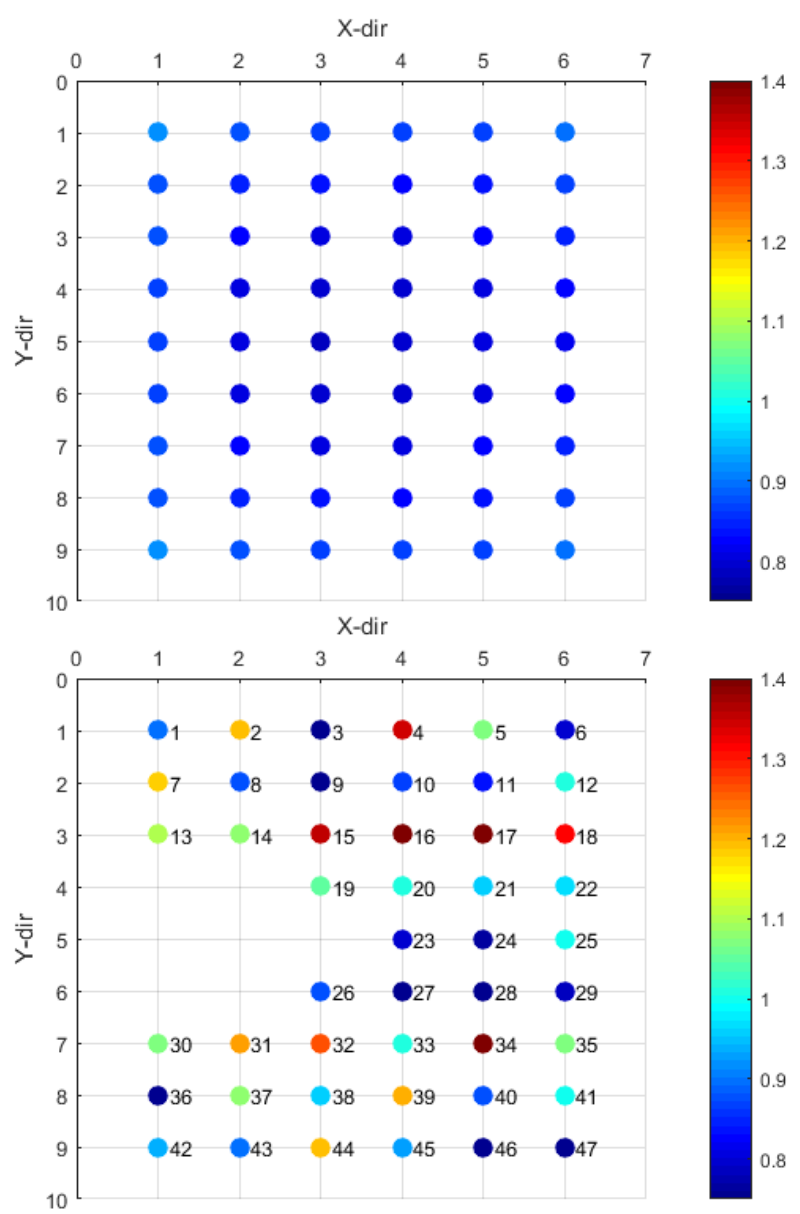

Figure 8. Top, precision of the design computations at $z=1$ in $[\mathrm{m}]$. Bottom, the estimated precision by the BlooLoc IPS in [m].

Overall, the design computations provide a good prediction model in order to determine whether a required precision and reliability can be acquired for any geometric configuration within a 3D space. However, to explain significant differences between the model results and the results of the practical experiment, the design computations will need to be elaborated further in order to account for reflections, materialistic properties, signal attenuation etc.

\subsection{Finding the optimal configuration}

The initial model requires a manual selection of beacon locations in order to compute the precision and reliability. However, the model can be transformed in a model selecting the optimal geometric configuration by assessing the precision results of millions of geometric configurations and a performance threshold (e.g. required precision) depending on the use case. In such a case, a customer could define several requirements or input parameters such as the dimensions of a 3D space, the number of available beacons, a performance threshold, and possible tag- and beacon locations. Subsequently, the model is able to find the optimal geometric configuration based on the users requirements and input parameters. Therefore, instead of assigning the 15 positions of the beacons, an infinite amount of possible beacon positions can be given as input parameters. For this example, the beacons could be deployed on 27 possible locations within a 10x10x5 theoretical space, see Figure 9. From Fig. 9 can be seen that there are 8 possible beacon locations at $z=1$ and another 8 possible beacon 
locations at $z=3$. Additionally, 11 possible beacon positions are distributed over the ceiling at $z=5$.

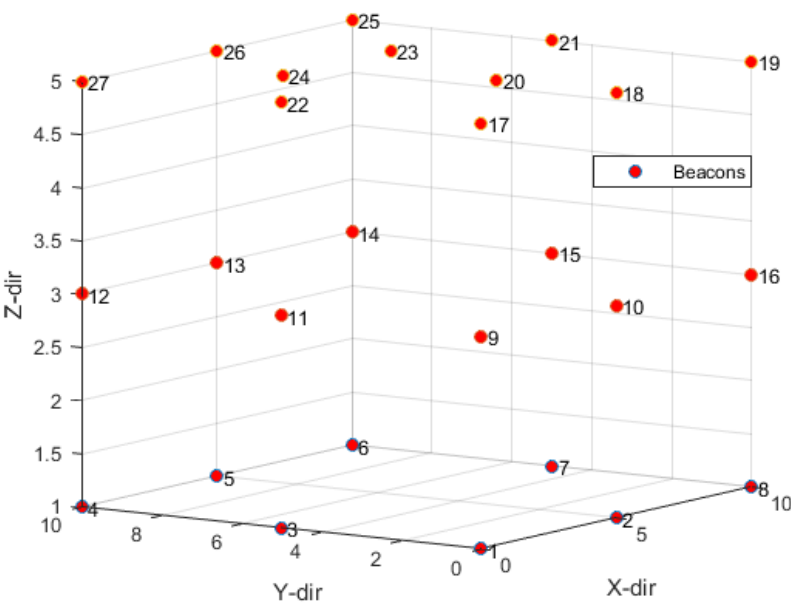

Figure 9. The 27 possible beacon locations for a 10x10x5 theoretical space.

Every geometry consists of 15 beacons which is the maximum amount of available beacons in this paper. The model will generate every possible combination for the 27 possible beacon locations with 15 beacon locations every run. Note that repetition of beacons is not allowed and that order of selection does not matter. In this case, the amount of possible combinations $C$ can be derived by using the factorial as follows

$$
C=\frac{n !}{r !(n-r) !}
$$

where $n$ represents the number of possible beacon locations and $r$ represents the amount of selected beacons. Initially, $n=$ 27 and $r=15$ would result in $C=17383860$ combinations or possible geometric configurations. However, the run time of the model is increases when $C$ increases and for $C=17383860$ the run time is too long for an efficient product. Therefore, the amount of combinations was reduced by distributing the 15 beacons over the three height levels, namely 4 beacons at $z=1,4$ beacons at $z=3$ and 7 beacons at $z=5$. The 8 possible beacon locations at $z=1$ will take 4 of the 15 beacons into account, resulting in $C=70$ combinations $(n=8, r=4)$. Likewise, the 8 possible beacon locations at $z=3$ take 4 of the 15 beacons into account, which also results in $C=70$ combinations. The 11 possible positions at the ceiling $(z=5)$ use the resulting 7 of the 15 beacons, which gives $C=330$ combinations ( $n=11, r=7$ ). The total amount of combinations is then reduced to $C_{\text {total }}=70 * 70 * 330=1617000$, which decreases the run time and makes the model more efficient and profitable.

In addition, a performance threshold (e.g. required precision) is used to filter the 1617000 possible geometries in order to find the optimal geometric configuration. The performance threshold is depending on the user requirements and thus on the use case. Furthermore, the performance threshold is used to filter all possible combinations of geometric configurations and to find the optimal solution. Assume a specific use case would require a precision (standard deviation) of 1 meter at both $z=1$ and $z=4$, the total precision at all possible user locations can be examined against the threshold $\left(\sigma_{T} \leq 1.0\right)$ for every individual geometric configuration out of the 1617000 possible geometries. Subsequently, the percentage of the precision satisfying the threshold at the user locations at $z=1$ and $z=4$ was computed and compared to the previous percentage in an iterative model. Eventually, the optimal solution was obtained by finding the geometry that corresponds with the highest percentage of the precision at the possible user locations satisfying the threshold. The threshold of $\sigma_{T} \leq 1.0$ resulted in a single solution with a percentage of $94,4 \%$ of the user locations at $z=1$ and $z=4$ satisfying the threshold. The geometric configuration and the precision results at $z=1$ and $z=4$ are depicted in Figure 10.

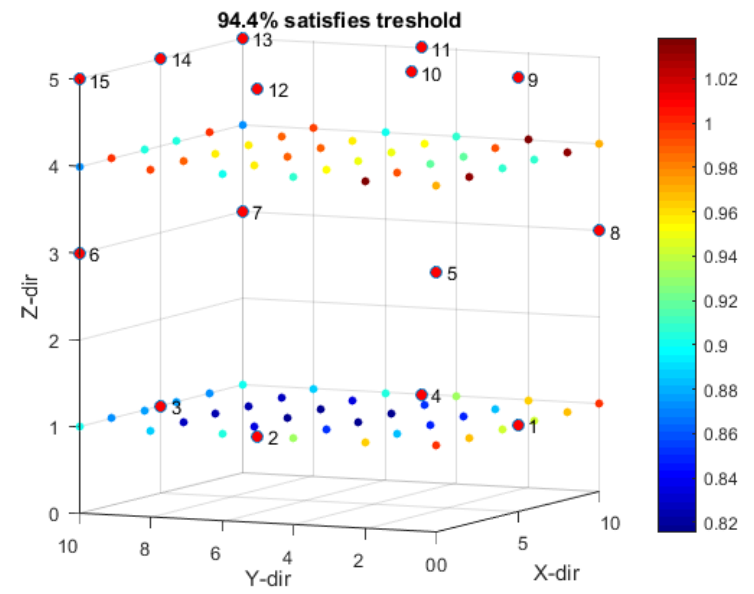

Figure 10. The optimal geometric configuration satisfying the threshold in terms of precision for $94.4 \%$ of the user locations.

3.3.1 Varying the threshold and number of beacons: The threshold is highly dependent on the use case and is therefore variable. Different thresholds result in different optimal geometric configurations. Therefore, the model performed multiple runs to examine the differences in optimal geometric configurations for various thresholds. The threshold starts at $\sigma_{T} \leq 1.0 \mathrm{~m}$ and decreases with intervals of 0.01 meter until the model has no possible solutions. Furthermore, the maximum amount of available beacons (15) was used for finding the first optimal geometric configuration. However, customers are not only interested in the optimal solution, but also in the optimal solution that reduces the costs while the solution still satisfies the performance threshold of the use case. If the optimal solution satisfies the performance threshold for less beacons, the total costs would reduce. Therefore, to investigate the optimal solution in terms of costs the amount of available beacons can be reduced. Consequently, two additional runs have been performed by the model. The model generates every possible combination for the same 27 possible beacon locations of Fig. 9, however this time with 14 available beacons and another run with 13 available beacons (beacon is subtracted from the beacons at the ceiling). Similar to the run with 15 beacons, repetition of beacons is not allowed and that order of selection does not matter.

To illustrate the effect of decreasing the amount of available beacons on the percentage of the user locations at $z=1$ and $z=4$ that satisfy the threshold, the results for 15,14 and 13 beacons are visualized in Figure 11. By decreasing the threshold, the percentage of the user locations satisfying the threshold decreases and the three graphs almost show a linear pattern. The difference between the graphs of 15 and 14 beacons is on average $13.6 \%$, whereas the graphs of 14 and 13 beacons differ on average 15.0 $\%$. The results can be used be used to determine how many beacons are needed for use cases with specific user requirements in 
terms of system performance. Therefore, the theoretical design computations help the customer in selecting the optimal geometry for a specified performance threshold and even show whether the same threshold is satisfied in case less beacons would be available. Eventually, the customer saves time and money in selecting the optimal configuration for his IPS and is able to get the required precision.

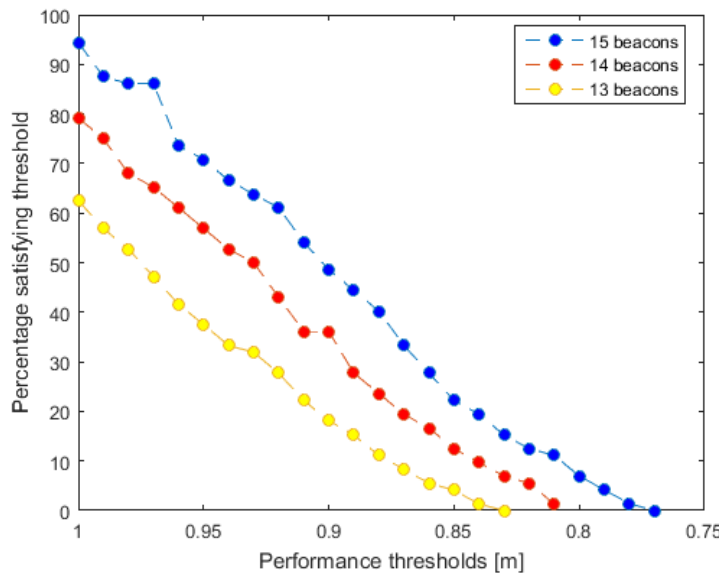

Figure 11. The percentage (\%) of the user locations satisfying the thresholds in terms of precision versus the performance thresholds for 15, 14 and 13 available beacons.

\section{CONCLUSIONS}

All in all, the design computations provide a good initial prediction model which is able to assess the quality of data with multiple hypothetical geometries for any 3D space. For any given set of beacon and receiver locations, the precision, internal- and external reliability can be determined in advance, saving time and money of the customer in the process to find the configuration that coincides with the best precision results. Besides determining the precision based on a set of beacon and receiver locations, the model is able to select the optimal geometric configuration based on a performance threshold (e.g. required precision). The performance threshold varies depending on the use case and the user requirements. Therefore, the amount of possible combinations in terms of 3D space, amount of available beacons, possible beacon locations, user tag locations and performance thresholds are limitless and the model can thus be used for all kind of applications. In addition, the design computations are not bound to a specific IPS and can be used for every IPS using transmitters and receivers for localization. However, some significant differences between the model results and the results of the practical experiment can only be explained physically, but were not implemented in the theoretical design computations yet. Therefore, the design computations will need to be elaborated further in order to account for reflections, materialistic properties, signal attenuation etc.

\section{FUTURE WORK}

Future work comprises adaptions to the model such that it can explain significant differences between the model and the measured data by including factors that influence the system performance in real life, such as materialistic properties, multipath (reflection) signal attenuation (refraction), NLOS etc. Eventually, a finalized model can be used as analysis tool for property owners. The model is able to recommend the users where to place the beacons such that their performance threshold in terms of precision is satisfied. The model would be dependent on several parameters that need to be provided by the user/customer. The parameters of interest would be the size of the property/3D space, performance threshold in terms of precision, amount of available beacons, possible user within the 3D space and the materialistic properties of the walls and objects (to account for reflection and refraction). Therefore, if a customer would provide these parameters, the model would be able to find the optimal solution and a recommendation for the customer could be made based on the results of the analysis tool.

\section{References}

Bhagwat, P., 2001. Bluetooth: technology for short-range wireless apps. IEEE Internet Computing 5(3), pp. 96-103.

BlooLoc, 2016. Blooloc products and technology. https://www.blooloc.com/ (15 Apr. 2017).

Callaerts, D., 2016. Yoobee, a sensor fusion platform for accurate indoor positioning. Guest lecture by BlooLoc at the course 'Positioning and Location Awareness (GEO3001)' at MSc track Geomatics, TU Delft.

Depsey, M., 2003. Indoor positioning systems in healthcare. $R a$ dianse Inc., White Paper.

Dursch, A., Yen, D. C. and Shih, D.-H., 2004. Bluetooth technology: an exploratory study of the analysis and implementation frameworks. Computer standards \& interfaces 26(4), pp. 263 277.

Faragher, R. and Harle, R., 2014. An analysis of the accuracy of bluetooth low energy for indoor positioning applications. In: Proceedings of the 27th International Technical Meeting of The Satellite Division of the Institute of Navigation (ION GNSS+ 2014), Tampa, FL, USA, Vol. 812

Gomez, C., Oller, J. and Paradells, J., 2012. Overview and evaluation of bluetooth low energy: An emerging low-power wireless technology. Sensors 12(9), pp. 11734-11753.

Langley, R. B., 1999. Dilution of precision. GPS world 10(5), pp. $52-59$

Mautz, R., 2012. Indoor positioning technologies. Habilitation thesis, ETH Zurich

Omre, A. H. and Keeping, S., 2010. Bluetooth low energy: wireless connectivity for medical monitoring. Journal of diabetes science and technology 4(2), pp. 457-463.

Spilker, J., 1996. Satellite constellation and geometric dilution of precision. Global Positioning System: Theory and applications. 1, pp. 177-208.

Teunissen, P., Simons, D. and Tiberius, C., 2004. Probability and observation theory.

van der Ham, M. F. S., Zlatanova, S., Verbree, E. and Voûte, R., 2016. Real time localization of assets in hospitals using quuppa indoor positioning technology. ISPRS Annals of Photogrammetry, Remote Sensing and Spatial Information Sciences IV-4/W1, pp. 105-110. 